\title{
Guía de práctica clínica: Depresión en adultos con problemas crónicos de salud
}

Depression in adults with a chronic physical health problems (NICE Clinical Guideline 91, october 2009)

Traducido con autorización del National Institute for Health and Clinical Excellence (NICE). Disponible en: http://www.nice.org.uk/nicemedia/live/12327/45865/45865.pdf

\section{Introducción}

La depresión es aproximadamente dos a tres veces más común en pacientes con un problema de salud o enfermedad crónica (como el cáncer, enfermedades del corazón o diabetes) que en las personas sin estos trastornos. Los problemas crónicos de salud pueden causar y agravar la depresión, y el tratamiento de la depresión en estos pacientes tiene el potencial de aumentar su calidad y su esperanza de vida. La presencia de una enfermedad puede complicar el reconocimiento y la evaluación de la depresión, debido a que algunos síntomas son comunes a ambos trastornos. Los síntomas asociados a la depresión, aun cuando estos se encuentren bajo un umbral diagnóstico pueden ser angustiantes y dis-

Enfoque de la intervención

PASO 4: depresión severa y compleja; con riesgo de vida; auto-abandono severo.

PASO 3: síntomas depresivos subumbral persistentes, o síntomas depresivos de leve a moderados con respuesta inadecuada a las intervenciones iniciales; depresión moderada a severa.

PAS0 2: síntomas depresivos subumbral persistentes; síntomas depresivos leves a moderados.

PAS0 1: todas las presentaciones conocidas o sospechadas de depresión. capacitantes, especialmente en pacientes con un problema crónico de salud. Por lo tanto, esta guía también se refiere a "los síntomas depresivos subliminales".

\section{Modelo de intervención escalonado}

Este modelo proporciona un marco para organizar la prestación de servicios, y ayuda a los pacientes, los cuidadores y los profesionales de la salud a identificar y acceder a las intervenciones más eficaces. La intervención menos intrusiva y más eficaz será siempre la primera en implementarse. Si una persona no se beneficia o no acepta una determinada intervención se debería ofrecer una intervención apropiada del siguiente escalón.

Naturaleza de la intervención

Medicación, intervenciones psicológicas de alta intensidad,

terapia electroconvulsiva, atención de crisis, tratamientos

combinados, multidisciplinarios e internación

Medicación, intervenciones psicológicas de alta intensidad, tratamientos combinados, cuidado conjunto y derivación para evaluación e intervenciones ulteriores.

Intervenciones psicológicas y psicosociales de baja intensidad, medicación y derivación para evaluación e intervenciones ulteriores.

Evaluación, apoyo, psico-educación, monitoreo activo y

derivación para evaluación e intervenciones ulteriores.

Severidad de la depresión

-Síntomas depresivos sub-umbral: menos de cinco síntomas.

-Depresión leve: los síntomas generalmente exceden por poco, si lo hacen, a los cinco síntomas necesarios para establecer el diagnóstico; provocan escasa repercusión funcional.

-Depresión moderada: los síntomas y el deterioro funcional resultan entre leves y severos.

-Depresión severa: gran cantidad de síntomas, con marcada repercusión funcional. Pueden coexistir o no con síntomas psicóticos.

\section{Paso 1: Identificando la depresión}

Preguntar al paciente potencialmente deprimido:

- Durante el último mes, ¿se ha sentido molesto frecuentemente por: sentirse desanimado, deprimido o desesperanzado? ¿tener poco interés o placer en hacer cosas?

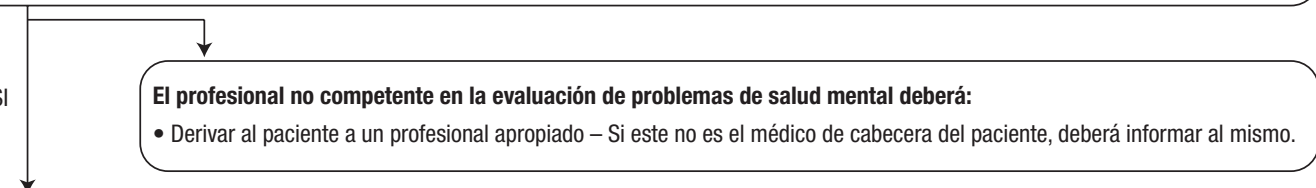

\footnotetext{
El profesional competente en la evaluación de problemas de salud mental deberá:

- Realizar tres preguntas adicionales para incrementar la precisión de la evaluación: durante el último mes, ¿se ha sentido frecuentemente molesto por: sentimientos de inutilidad; poca concentración; o pensamientos de muerte?

- Realizar una evaluación ampliada, que no se base solamente en la recopilación de síntomas, y que tenga en cuenta: el grado de compromiso funcional o discapacidad asociada; y la duración del episodio.

- Considerar el rol del problema concomitante de salud, y la prescripción o uso de cualquier medicación para la depresión.

- Corroborar que se este proporcionando el tratamiento óptimo para el problema de salud asociado, buscando la intervención de especialistas de ser necesario.

- Explorar como pueden haber afectado en el desarrollo, curso y severidad de la depresión: los antecedentes de depresión (incluidos los tratamientos implementados); las comorbilidades psiquiátricas u otros problemas de salud; las relaciones interpersonales, condiciones de vida y el aislamiento social.

- En pacientes con dificultades idiomáticas o de comunicación, considerar el uso de herramientas de detección de estrés y/o indagar con algún familiar o cuidador acerca de los síntomas.
} 


\section{Paso 2}

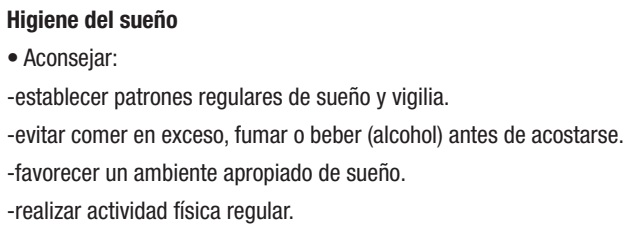

\section{Monitoreo activo}

- Para aquellos pacientes que podrían recuperarse sin intervención formal alguna, o aquellos con depresión leve o que no desean ninguna intervención o tienen síntomas depresivos subumbral y solicitan alguna intervención:

-discutir el problema y las preocupaciones relacionadas.

-brindar información sobre depresión.

-establecer una visita de seguimiento, habitualmente dentro de las dos semanas próximas.

-establecer contacto si el paciente no concurre a las citas de control.

\section{Intervenciones psicosociales de baja intensidad}

- Considerar ofrecer una o mas de las siguientes a los pacientes con síntomas depresivos subumbral persistentes, depresión leve a moderada, 0 a los pacientes con depresión sub-umbral en quienes se complejiza la atención de su problema crónico asociado: -un programa de actividad física (adaptada al problema de salud del paciente).

-la participación en reuniones grupales de pacientes con un mismo problema crónico de salud. -intervenciones de autoayuda basadas en principios de terapia cognitivo conductual (TCC).

-TCC computarizada.

Para mas detalles sobre estas últimas intervenciones, remitirse a la sección 1.4.2 en URL www.nice.org.uk/CG91niceguideline

\section{Tratamiento farmacológico}

- No utilice tratamientos antidepresivos rutinariamente para tratar síntomas depresivos sub-umbral o síntomas depresivos leves, pero considere estos fármacos en pacientes con: -historial de depresión moderada a severa, 0

-depresión leve que complejiza el tratamiento del problema crónico asociado, 0

-presentación inicial de síntomas depresivos sub-umbral de al menos dos años de evolución, 0 -síntomas depresivos sub-umbral o depresión leve que persisten luego de otras intervenciones.

- No prescribir o desaconsejar el uso de hipérico (planta de San Juan). Explicar que es difícil establecer equivalencias en la potencia de las preparaciones disponibles y la posibilidad de que se produzcan interacciones serias con otras medicaciones.

\section{Paso 3}

Opciones terapéuticas

\begin{tabular}{l|l}
\multicolumn{1}{c|}{ Población diana } & \multicolumn{1}{c}{ Opción terapéutica } \\
\hline $\begin{array}{l}\text { - Pacientes con síntomas depresivos subumbral persistentes o depresión leve a } \\
\text { moderada que no se han beneficiado de una intervención psicosocial de baja } \\
\text { intensidad }\end{array}$ & $\begin{array}{l}\bullet \text { Un antidepresivo, 0 } \\
\bullet \text { Una intervención psicológica de alta intensidad (TCC individual, grupal o de pareja). } \\
\text { ver www.nice.org.uk/CG91niceguideline }\end{array}$ \\
\hline - Pacientes que se presentan inicialmente con depresión moderada & $\bullet$ Una intervención psicológica de alta intensidad (TCC individual, grupal o de pareja). \\
\hline - Pacientes que se presentan inicialmente con depresión severa & $\bullet$ Considerar indicar tanto TCC como antidepresivos \\
\hline
\end{tabular}

- Al considerar una intervención, tener en cuenta:

-la duración del episodio y la trayectoria de los síntomas.

-antecedentes previos de episodios depresivos y respuesta al tratamiento.

-probabilidad de adherencia al tratamiento y efectos adversos potenciales.

-evolución y tratamiento del problema crónico de salud asociado.

-preferencias del paciente en cuanto a tratamiento.

Pacientes con depresión moderada a severa que no han respondido a las intervenciones iniciales

- Considerar el cuidado colaborativo o conjunto de los pacientes cuya depresión no ha respondido a las intervenciones psicológicas de alta intensidad, tratamiento farmacológico o la combinación de ambos.

- El cuidado colaborativo deberá incluir la supervisión de un especialista en salud mental experimentado.

ver detalles en www.nice.org.uk/CG90quickrefguide 


\section{Paso 4}

- Las intervenciones pueden incluir medicación, intervenciones psicológicas de alta intensidad, terapia electroconvulsiva u otras terapias físicas, tratamientos combinados,

equipos de resolución de crisis o de manejo domiciliario, e internación de pacientes (para mas detalles ver www.nice.org.uk/CG90quickrefguide)

- Si el tratamiento es proporcionado por servicios especializados en salud mental en pacientes con cuadros complejos y severos de depresión, deberá existir un trabajo

conjunto estrecho con el equipo que atiende el problema crónico de salud asociado.

\section{El cuidado de los pacientes con depresión}

\section{Principios de la atención}

- Tenga en cuenta que el diagnóstico de depresión puede asociarse con estigmatización y discriminación. Se deberá asimismo asegurar al paciente que se respeta su confidencialidad, privacidad y dignidad.

- Sea sensible a la diversidad cultural, étnica y religiosa; y este atento a variaciones en la presentación de los síntomas.

- Esté atento a la posibilidad de trastornos cognitivos asociados; considere la consulta diagnóstica especializada.

- Cuando la atención de un paciente es compartida entre ámbitos de atención primaria y secundaria, involucre al médico de cabecera. Comparta el plan de tratamiento con el paciente y (si procede) con su familia o cuidador.

- Si el problema crónico de salud del paciente limita su capacidad de participar en un tratamiento psicosocial o psicológico preferido, tenga en cuenta las alternativas como el tratamiento farmacológico o la administración de intervenciones por teléfono (si el contacto personal no es posible).

- Sea consciente de las posibles interacciones de los antidepresivos con los medicamentos recetados para los problemas crónicos de salud.

- Todas las intervenciones deben ser prestadas por profesionales competentes.

\section{Evaluación del riesgo y monitoreo}

- Si un paciente presenta un riesgo inmediato considerable para ellos mismos u otros, este deberá ser derivado en forma urgente a servicios especializados de salud mental.

- Asesore al paciente, su familia o cuidador sobre los siguientes puntos, y asegúrese de que estos sabrán cómo buscar ayuda rápidamente si es necesario:

-el potencial aumento, al inicio del tratamiento, de síntomas como agitación, ansiedad e ideación suicida; se deberá investigar activamente estos síntomas y reevaluar el tratamiento del paciente ante la aparición de agitación marcada y/o prolongada.

-la necesidad de estar atentos a los cambios de humor, negativismo, desesperanza e ideación suicida, sobre todo al iniciar o modificar el tratamiento y en los momentos de mayor estrés - asesorar a los pacientes a contactar a su médico en caso de preocupación.

- Pregunte siempre de manera directa a un paciente con depresión y un problema crónico de salud asociado sobre ideación o intentos suicidas.

- Si se considera que el paciente se encuentra en riesgo incrementado de suicidio, considerar:

-la proporción de un mayor apoyo, como el contacto más frecuente.

-la derivación a servicios especializados de salud mental.

\section{Antidepresivos}

Elección de antidepresivos

- Al elegir un antidepresivo, tenga en cuenta lo siguiente: -la presencia de otros problemas crónicos de salud. -los efectos adversos (que podrían impactar en el problema crónico de salud asociado).

-que actualmente no existe evidencia que apoye la elección de una droga determinada en función de un problema crónico de salud específico.

- Este atento a las interacciones medicamentosas y:

-consulte la tabla de interacciones medicamentosas en el apéndice 16 de la guía completa

(ver www.nice.org.uk/CG91fullguideline).

-busque ayuda especializada si existe incertidumbre.

-de ser necesario, derive al paciente a servicios especializados de salud mental para continuar con la prescripción.

- Prescriba un IRSS como primera elección - considere citalopram o sertralina.

- No deberá prescribirse dosuleptin.

- La prescripción de IMAO o antidepresivos asociados a litio debería ser realizada solamente por especialistas en salud mental.

- Tenga en cuenta la posibilidad de sobredosis al elegir un antidepresivo en un paciente con riesgo incrementado de suicidio. Tenga en cuenta que:

-comparado con otros antidepresivos equipotentes, la venlafaxina se asocia a mayor riesgo de muerte en caso de sobredosis. -los antidepresivos tricíclicos, a excepción de lofepramina, se asocian con el mayor riesgo de ante una sobredosis.

\section{Tratamiento}

- Explore cualquier preocupación del paciente relacionada con el uso de medicación, incluyendo:

-el desarrollo gradual del efecto antidepresivo pleno.

-la importancia de tomar la medicación de la forma como se prescribió y la necesidad de continuar el tratamiento mas allá de la remisión.

-los efectos adversos potenciales e interacciones medicamentosas. -la naturaleza y riesgo de síntomas que llevan a la discontinuación.

-el hecho que el tratamiento no conlleva riesgo de adicción.

- No prescriba antidepresivos en dosis subóptimas.

- Citar a los pacientes en los que se inició el tratamiento antidepresivo y no se encuentran bajo riesgo incrementado de suicidio a las dos semanas. Cítelos luego a control, cada dos a cuatro semanas durante los primeros tres meses, y luego a intervalos más prolongados en caso de buena respuesta.

- En los pacientes de mayor riesgo la cita inicial luego del inicio del tratamiento farmacológico se recomienda dentro de la primera semana.

- Si el paciente desarrolla efectos adversos de manera temprana, informe y considere lo siguiente:

-monitoree los síntomas de manera estrecha, si estos son leves y resultan aceptables para el paciente.

-suspenda o rote a un antidepresivo diferente según la preferencia del paciente, o

-realice un tratamiento corto concomitante (no mayor a dos semanas) con una benzodiazepina si resultan problemáticos la aparición de síntomas como ansiedad, agitación o insomnio, excepto en los pacientes con síntomas crónicos de ansiedad; sea precavido en los pacientes con riesgo de caídas. 
Discontinuación de los antidepresivos

- Normalmente, reduzca gradualmente la dosis durante cuatro semanas (esto no es necesario con fluoxetina). Reduzca la dosis durante periodos mas prolongados cuando se utilizan drogas con vida media mas corta (ej. paroxetina o venlafaxina). - Aconseje al paciente a consultar si experimenta síntomas significativos de discontinuación.

\section{Interacciones de los IRSS con otras medicaciones}

\begin{tabular}{|c|c|}
\hline Medicación para problemas crónicos de salud & Antidepresivo(s) recomendados \\
\hline Anti-inflamatorios no esteroides (AINE) & $\begin{array}{l}\text { - No ofrezca IRSS de manera habitual, aunque si no se pueden identificar alternativas, recomendar } \\
\text { gastroprotectores (inhibidores de la bomba de protones) en forma conjunta. } \\
\text { - Considerar mianserina, mirtazapina, moclobemida, reboxetina o trazodona. }\end{array}$ \\
\hline Warfarina y heparina & $\begin{array}{l}\text { - No ofrezca habitualmente IRSS. } \\
\text { - Considere mirtazapina (tenga en cuenta que cuando se co-administran el rango internacional normatizado } \\
\text { puede incrementarse en forma leve). }\end{array}$ \\
\hline Aspirina & $\begin{array}{l}\text { - Utilice los IRSS con precaución, aunque si no se pueden identificar alternativas, recomiende el uso de } \\
\text { gastroprotectores en forma conjunta. } \\
\text { - Cuando se utiliza aspirina como único agente, considere el uso de mianserina, trazodona, o reboxetina. } \\
\text { - Considere el uso de mirtazapina. }\end{array}$ \\
\hline Triptanes (p/migraña); IMA0 (ej. selegilina o rasegilina) & $\begin{array}{l}\text { - No ofrezca IRSS } \\
\text { - Considere mirtazapina, trazodona, mianserina o reboxetina. }\end{array}$ \\
\hline Teofilina, clozapina, metadona o tizamidina & $\begin{array}{l}\text { - No ofrezca fluvoxamina } \\
\text { - Ofrezca sertralina o citalopram }\end{array}$ \\
\hline Flecainida o propafenona & $\begin{array}{l}\text { - Ofrezca sertralina como primera elección } \\
\text { - Considere alternativamente mirtazapina o moclobemida }\end{array}$ \\
\hline Atomoxetina & $\begin{array}{l}\text { - No ofrezca fluoxetina o paroxetina } \\
\text { - Ofrezca un IRSS diferente }\end{array}$ \\
\hline
\end{tabular}

IRSS: inhibidores de la recaptación de serotonina. IMAO: inhibidores de la mono-amino-oxidasa.

Nota: las guías NICE son desarrolladas para su aplicación dentro del Servicio Nacional de Salud (sigla en ingles NHS) en el Reino Unido. EI NICE no toma responsabilidad alguna por la traducción al español aquí publicada de la presente guía.

\section{Comentario}

La presente guía NICE intenta sistematizar el manejo y tratamiento de pacientes adultos con depresión y un problema crónico (físico) de salud asociado, a partir de un enfoque escalonado; tratando así de ofrecer al médico y al paciente, múltiples alternativas terapéuticas, desde aquellas mas simples y efectivas hasta las mas complejas; la misma otorga una

Maximiliano Smietniansky [ Servicio de Clínica Médica del Hospital Italiano de Buenos Aires maximiliano.smietniansky@hospitalitaliano.org.ar ]

Recibido el 21/04/2010 y aceptado el 15/01/2011

Smietniansky M. Depresión en adultos con problemas crónicos de salud asociados. Evid Act Pract Ambul 14(2) 71-74. Traducido y comentado de: Depression in adults with a chronic physical health problema (NICE Clinical Guideline 91, october 2009). Disponible en: http://www.nice.org.uk/nicemedia/live/12327/45865/45865.pdf gran importancia a todos los tratamientos no farmacológicos dejando para cuadros más severos o complejos, la opción farmacológica. Esto, así establecido, constituye un marco de referencia para dar fundamento y orden al criterio médico, pero de ningún modo lo excluye; así como tampoco los múltiples aspectos psicológicos, familiares y sociales, que atraviesan al paciente y a su enfermedad. 